\title{
Déplacements des spermatozoïdes dans l'oviducte chez la poule et la dinde
}

\author{
JP Brillard \\ INRA, Station de recherches avicoles, I 37380 Nouzilly, France
}

(Reçu le 13 juin 1989; accepté le 23 janvier 1990)

\begin{abstract}
Résumé - Des progrès récents dans la compréhension des mécanismes de stockage et d'évacuation des spermatozoïdes présents dans les glandes utéro-vaginales des oiseaux ont montré que le stockage est réalisé en 2-3 jours chez la poule ou 1-5 jours chez la dinde après insémination intravaginale et non en quelques heures comme cela avait été avancé jusqu'alors. Le fait que les spermatozoïdes puissent être retrouvés dans n'importe quel segment de l'oviducte au cours du cycle de formation de l'œuf rend peu vraisemblable l'idée selon laquelle leur évacuation depuis les glandes utéro-vaginales serait en relation avec le cycle de formation de l'œuf. Par contre la perte progressive par les spermatozoïdes de leur capacité à s'agglutiner pourrait être impliquée dans ce mécanisme.
\end{abstract}

spermatozoïde / oviducte / stockage in vivo / oiseau / transport

Summary - Sperm transport through the avian oviduct. Recent studies of the storage and release of spermatozoa from utero-vaginal glands in birds have shown that: following intra-vaginal insemination, storage is completed within 2-3d (domestic hen) or 1-5d (turkey) and not within a few minutes or hours as previously described; as spermatozoa can be recovered from any segment of the oviduct during the egg formation cycle, it seems unlikely that sperm release from the uterovaginal glands is directly dependent upon the egg formation cycle. The progressive inability of spermatozoa to agglutinate may be part of this mechanism.

spermatozoa / oviduct / in vivo storage / bird / transport

\section{INTRODUCTION}

Chez les oiseaux, l'existence d'un stockage prolongé des spermatozoïdes dans la jonction utéro-vaginale ainsi que les sécrétions par l'oviducte des constituants de l'œuf (albumen, membranes coquillières, coquille...) ont des conséquences importantes sur les déplacements des gamètes mâles vers la zone de fécondation située dans la partie haute du pavillon (Olsen et Neher, 1948). En schématisant, les spermatozoïdes sont déposés à l'entrée du vagin puis gagnent les glandes utéro- vaginales où ils séjournent pendant une durée qui varie, selon les espèces, de quelques jours à quelques semaines. Après avoir quitté ces glandes, ils doivent accéder à la partie haute du pavillon pour participer à la fécondation de l'œuf. Comment se réalisent ces déplacements ? En particulier, la mobilité propre des spermatozoïdes est-elle suffisante ? Existe-t-il des périodes privilégiées du cycle de formation de l'œuf qui favorisent ou défavorisent leur remontée ? Nous rapportons ici les éléments bibliographiques dont nous disposons à ce jour pour tenter de répondre à chacune de ces questions. 


\section{DÉPLACEMENT DES SPERMATO- ZOIDES DANS L'OVIDUCTE}

La migration des spermatozoïdes dans les voies génitales femelles met en jeu des mécanismes différents selon qu'il s'agit des zones situées en deçà ou bien audelà de la jonction utérovaginale. C'est ainsi que leur motilité propre est nécessaire pour la traversée de la région vaginale (transport actif) alors que cette motilité n'est pas indispensable pour le franchissement de la partie supérieure de l'oviducte (transport passif). En effet, des spermatozoïdes morts déposés à l'entrée du vagin n'atteignent jamais les glandes de la jonction utérovaginale, mais des spermatozoïdes morts ou privés de leur flagelle, placés dans l'utérus, sont retrouvés dans le pavillon chez la poule (Allen et Grigg, 1957) et la dinde (Verma et Cherms, 1966). Le battement des cils qui tapissent l'ensemble de la muqueuse oviducale ne peut être, à lui seul, responsable de la migration passive des spermatozoïdes vers l'ovaire car, au moins dans l'utérus et dans l'isthme, il déplace le fluide utérin en sens opposé (Mimura, 1941). Allen et Grigg (1957) émettent l'hypothèse d'un transport passif par les contractions spasmodiques de l'oviducte, alors qu'EI Jack et Lake (1967) évoquent la possibilité d'une remontée active reliée aux variations de composition du fluide utérin au moment de l'oviposition (celle-ci, chez la poule, précède de 25 minutes environ l'ovulation suivante). Pourtant la remontée des spermatozoïdes est possible en dehors de la période d'oviposition (Burke et al, 1969; Bakst, 1988). De plus, la migration de particules de carbone dans différentes régions de l'oviducte de dindes 5-10 min puis 50-55 min après l'oviposition montre que le sens de déplacement des particules varie selon la région de l'oviducte concernée et selon le stade du cycle de forma- tion de l'œuf (Fujihara et al, 1983). S'il est donc établi que la motilité des spermatozoïdes chez les oiseaux est nécessaire pour traverser la région vaginale, il n'est pas possible à ce jour de préciser quelles sont les causes de leur migration dans la partie supérieure de l'oviducte. Une seule des hypothèses avancées n'a pas été invalidée : elle concerne l'action des contractions oviducales. Elle impliquerait cependant qu'aux contractions péristaltiques qui contrôlent le déplacement de l'œuf se superposent d'autres contractions organisées en direction opposée... ce qui n'a jamais été démontré. Une autre voie de recherche mériterait d'être explorée : elle concerne les déplacements de fluide utérin au cours du cycle de ponte. Ce fluide paraît être sécrété en quantité et en durée excessives par rapport aux besoins de l'œuf pendant la phase d'hydratation de l'albumen, ou "plumping" (Hoover et Smith, 1958). ॥ n'est pas impossible qu'une partie de ce liquide, s'il n'est pas immédiatement résorbé, parvienne jusqu'à l'infundibulum et entraine avec lui des spermatozoïdes libérés par les glandes utéro-vaginales.

\section{CINÉTIQUE DU DÉPLACEMENT DES SPERMATOZOÏDES DANS L'OVIDUCTE}

Le temps nécessaire pour que les spermatozoïdes traversent l'oviducte est fort mal connu. Par exemple, chez la poule, il varie de $1 \mathrm{~h}$ (Allen et Grigg, 1957) à $24 \mathrm{~h}$ (Crew, 1926). Pour ces derniers auteurs ainsi que pour Bobr et al (1964), l'utérus - et à fortiori la jonction utéro-vaginale - est atteint en moins d'un quart d'heure. Toutefois, ces travaux ne tiennent compte que des migrations des spermatozoïdes les plus avancés : chez la dinde en ponte, leur présence est bien observée dans les glandes utérovaginales 90 min seulement après insémination, mais leur concentration y est bien 
plus élevée 5 j plus tard (Verma et Cherms, 1965), le maximum étant observé dans cette espèce, comme chez la poule, 3 j seulement après insémination (Prasad, 1967). Mais ces valeurs semblent dépendre de l'état physiologique des animaux, car chez des dindes non pondeuses, le stockage des spermatozoïdes dans les glandes est plus rapide ( $1 \mathrm{j}$ ) que celui observé chez des dindes en ponte : 5 j selon Mclntyre (1984). Chez la poule, nos propres résultats (Brillard, 1989a et tableau I) portent sur 2 souches de poules pondeuses (Isabrown) ou reproductrices (Vedette) inséminées en cours de saison de ponte (âges étudiés : 30-32 sem et 58-60 sem) avec des doses modérées $\left(60 \times 10^{6}\right)$ ou élevées $\left(240 \times 10^{6}\right)$ de spermatozoïdes. Les poules ont été abattues entre $1 \mathrm{~h}$ et $21 \mathrm{j}$ après insémination. Les premiers spermatozoïdes ont bien atteint les glandes utéro-vaginales dès la première heure suivant l'insémination de certains groupes d'oiseaux, mais à ce stade, la présence de spermatozoïdes n'est observée que dans 0,3 à $11,2 \%$ de ces glandes. Le stockage maximum $(21,9$ à $52,7 \%$ des glandes sont concernées, selon les lots) n'intervient que 2 jours après la mise en place du sperme dans 6 des 8 combinaisons expérimentales. Tous les facteurs testés : souche de poule, dose, âge et délai après insémination ont une action significative sur le stockage $(P<0,05)$, le facteur souche de poule étant le plus important dans nos conditions d'expérience. II paraît donc acquis que le stockage s'effectue progressivement en $2 \mathrm{j}$ environ chez la poule et, selon les auteurs en 1 à 5 j chez la dinde,. Ainsi, la mise en place du sperme dans les glandes de la jonction utéro-vaginale est plus longue que les quelques minutes d'abord avancées (Allen et Grigg, 1957; Bobr et al, 1964). Ceci implique que le passage d'un ou plusieurs cufs dans la lumière vaginale n'est pas incompatible avec la survie de spermatozoïdes non encore stockés dans cette zone. Cette observation est confirmée par la présence de nombreux spermatozoïdes dans la lumière de la région utérine (Burke et Ogasawara, 1969; Howarth, 1971) et

Tableau I. Évolution du pourcentage de sections transversales de glandes utéro-vaginales contenant des spermatozoïdes après l'insémination intra-vaginale de $60 \times 10^{6}$ ou $240 \times 10^{6}$ spermatozoïdes chez des poules reproductrices naines ou des poules pondeuses. (4 à 7 femelles/traitement). Pour un croisement, un âge et une dose donnés, les maxima (soulignés) ont été estimés par comparaison des moyennes entre groupes, après transformation (arcsin $\sqrt{\text { ) }}$ des pourcentages (lots sans résultat après 1 ou $6 \mathrm{~h}$ ) ou après addition séquentielle des jours et comparaison des coefficients de corrélation des régressions (autres lots).

Poules reproductrices naines

Jeunes (30-32 sem) Âgées $(58-60 \mathrm{sem})$

$60 \times 10^{6} 240 \times 10^{6} \quad 60 \times 10^{6} 240 \times 10^{6}$
Poules pondeuses

Jeunes (30-32 sem) Âgées (58-60 sem)

$60 \times 10^{6} 240 \times 10^{6} 60 \times 10^{6} 240 \times 10^{6}$

\begin{tabular}{|c|c|c|c|c|c|c|c|c|}
\hline $1 \mathrm{~h}$ & - & - & 5,5 & 11,2 & 8,7 & 0,3 & - & - \\
\hline $6 \mathrm{~h}$ & - & - & 13,1 & 12,8 & 11,2 & 36.9 & - & - \\
\hline $1 j$ & 11,8 & 24,1 & 14,5 & 21.9 & 18,0 & 32,9 & 17,0 & 23,0 \\
\hline $2 \hat{j}$ & 22.2 & 29.5 & 224 & 13,7 & 40.3 & 36,8 & 36.9 & 527 \\
\hline $4 j$ & 14,8 & 20,3 & 7,7 & 15,2 & 16,1 & 31,6 & 10,5 & 29,8 \\
\hline $7 \hat{j}$ & 8,5 & 9,1 & 9,7 & 12,4 & 27,2 & 36,6 & 11,7 & 9,9 \\
\hline $14 j$ & 3,9 & 7,8 & 3,8 & 6,0 & 12,4 & 16,9 & 2,8 & 4,4 \\
\hline $21 j$ & 0,5 & 4,4 & 2,1 & 0,7 & 7,3 & 10,6 & 0,3 & 7,0 \\
\hline
\end{tabular}


vaginale (Bakst, 1988; Brillard et Bakst, observations personnelles) pendant les premiers jours qui suivent l'insémination.

\section{MODE DE REMPLISSAGE DES GLANDES UTÉRO-VAGINALES}

Le remplissage des glandes se réalise progressivement et commence par celles situées dans la région la plus proche du vagin, les dernières remplies étant situées près de l'utérus (Verma et Cherms, 1965). Quand plusieurs inséminations successives ont lieu, les spermatozoïdes semblent se déposer en couches stratifiées. Ainsi, chez la poule, l'insémination successive de spermatozoïdes marqués au tritium puis non marqués montre, selon cet auteur, l'existence d'un dépôt séquentiel dans les glandes (Van Krey et al, 1981). En outre, la double insémination (à $4 \mathrm{~h}$ d'intervalle) de poules avec du sperme issu de mâles porteurs ou non du gène de nanisme $d w$ révèle que plus de $75 \%$ des poussins éclos sont issus d'œuts fécondés par la dernière insémination, ce qui semble indiquer que les derniers spermatozoïdes entrés dans les glandes sont aussi les premiers à en être sortis Compton et al, 1981). Une approche expérimentale comparable conduit aux mêmes conclusions chez la dinde que chez la poule (Christensen, 1981). Mais l'hypothèse d'une véritable stratification des spermatozoïdes dans les glandes utérovaginales reste très fragile car les seules observations directes dont nous disposons (Van Krey et al, 1981) sont difficiles à interpréter à partir des photographies publiées par les auteurs. Une alternative à l'insémination séquentielle des œufs à partir de spermatozoïdes stratifiés dans les glandes pourrait impliquer les spermatozoïdes stockés dans la lumière oviducale (voir paragraphe précédent), les premières fécondations étant alors proportionnelles au nombre de gamètes «libres" porteurs d'un caractère donné.

\section{STOCKAGE DES SPERMATOZOÏDES}

II est généralement admis qu'après avoir pénétré dans les glandes utéro-vaginales, les spermatozoïdes sont immobilisés (Bakst et Richards, 1985) et s'agglutinent : (poule : Van Krey et al, 1981) (dinde : Schuppin et al, 1984). Cette immobilisation temporaire serait une nécessité biologique qui, en épargnant la faible réserve d'énergie dont disposent les gamètes mâles, leur permet de l'utiliser plus tard lors de la fécondation. Elle constituerait le mécanisme qui contrôle leur stockage prolongé in vivo (Van Krey et al, 1981). S'il paraît possible que l'acide sialique présent à la surface des spermatozoïdes contribue à leur agglutination (Froman et Engel, 1989), certains cations tels que le zinc et le calcium pourraient être impliqués dans le contrôle réversible de la motilité, le calcium stimulant l'activité métabolique des spermatozoïdes et le zinc la déprimant. L'action stimulante du calcium, déjà décrite chez d'autres espèces (Garbers et Kopf, 1980; Crichton et al, 1982) a été confirmée dans le plasma séminal du coq (Ashizawa et Wishart, 1987). L'action inhibitrice du zinc sur la motilité s'exerce par l'intermédiaire d'une diminution de la consommation d'oxygène par les spermatozoïdes qui a pu être mise en évidence in vitro chez le dindon (Bakst, 1985).

\section{LIBÉRATION DES SPERMATOZOIDES À PARTIR DES GLANDES UTÉRO-VAGINALES}

Le déplacement des spermatozoïdes depuis la jonction utérovaginale jusqu'à l'in- 
fundibulum n'est possible qu'à certaines étapes du cycle de formation de l'œuf, car pendant les heures de déposition d'albumen, le magnun reste infranchissable (Mimura, 1939; Howarth, 1971; Bakst, 1981). En revanche, la présence d'un œuf calcifié dans l'utérus ne constitue pas un obstacle majeur à leur remontée chez la poule (Bobr et al, 1964; Burke et Ogasawara, 1969) et la dinde (Howarth, 1971). L'hypothèse a été avancée (Grigg, 1957) d'une décharge ponctuelle des spermatozoïdes par les glandes utéro-vaginales en liaison avec l'oviposition seule (effet mécanique de l'œuf par pression des glandes) ou en liaison avec l'oviposition et/ou l'ovulation suivante (Bobr et al, 1964). Toutefois la libération des spermatozoïdes stockés ne peut être provoquée chez des poules en ponte injectées de plusieurs types d'hormones ( $\mathrm{LH}$, progestérone, extraits hypophysaires, PMSG, ocytocine et vasopressine : Van Krey, 1964; Compton et Van Krey, 1979). Ces auteurs concluent à l'absence de relation entre la décharge des spermatozoïdes par les glandes et un quelconque phénomène en liaison directe avec le cycle de formation de l'œuf. De même, les nombres de spermatozoïdes présents dans les échantillons de fluide utérin collectés chez des poules en ponte $5 \mathrm{~h}$ avant jusqu'à $5 \mathrm{~h}$ après l'oviposition ne montrent aucune variation liée à la période de prélèvement; l'évacuation des spermatozoïdes vers la lumière oviducale semble donc indépendante de l'ovulation et/ou de l'oviposition (Burke, 1968; Burke et Ogasawara, 1969; Burke et al, 1972). Enfin, chez la dinde en ponte des spermatozoïdes sont présents dans toutes les parties luminales de l'oviducte, quel que soit le stade du cycle de formation de l'œuf (Bakst, 1981).

Nos propres résultats chez la poule en ponte sont aussi en faveur d'une libération prolongée (quelques $h$ ) plutôt que ponctuelle, des spermatozoïdes (Brillard, 1989b et tableau II). En effet, la probabilité de rencontre des spermatozoïdes avec l'œuf dans l'infundibulum (siège de la fécondation) est proche de $100 \%$ pendant la semaine qui suit l'insémination, car la fertilité des œufs est de l'ordre de $95 \%$ ou plus pendant cette période. En l'absence de stockage infundibulaire (Bobr et al, 1964), ceci suggère que les gamètes mâles ayant quitté la jonction utéro-vaginale traversent en continu l'infundibulum quel que soit le moment de l'ovulation, et qu'il y ait eu ou non oviposition.

Tableau II. Nombres calculés de spermatozoïdes retrouvés par lavage en continu de la cavité abdominale de poules inséminées par voie intramagmale. Insémination de $200 \times 10^{6}$ spermatozoïdes/ femelle; comptages réalisés sur filtres Millipore (maille 0,25 $\mu \mathrm{m}$ ).

Délai après insémination

$$
\text { Poule } 1 \stackrel{\text { Nombre de spermatozoïdes }\left(10^{6}\right)}{\text { Poule } 2} \text { Poule } 3
$$

\begin{tabular}{rlcl}
15 & 20 & 0,1 & 0,1 \\
30 & 31 & 0,7 & 4 \\
45 & 39 & 35 & 20 \\
60 & 42 & 46 & 28 \\
90 & 46 & 65 & 46 \\
120 & 49 & 68 & 55 \\
150 & 52 & 70 & 58 \\
180 & 55 & 72 & 60 \\
\hline
\end{tabular}


Chez les poules en pause naturelle ou provoquée, les résultats sont plus contradictoires. Ainsi, chez celles dont les ovulations ont été bloquées durablement par injection de PMSG, Burke et al (1969) observent une diminution, mais non l'absence d'évacuation des spermatozoïdes stockés dans les glandes utéro-vaginales, ce qui tend à confirmer les résultats précédents. Mais Bushman et al (1985) notent qu'après traitement à la PMSG, les glandes utéro-vaginales ne se vident pratiquement pas, au moins pendant les 12 premiers jours qui suivent l'insémination. Enfin, Bilgili et al (1984) ne constatent aucune différence de fertilité entre des poules inséminées en pré-pause (oviposition sans ovulation ultérieure), en pause (pas d'ovulation) ou en non-pause (avec ovulation et oviposition). Ce dernier résultat indique qu'un nombre de spermatozoïdes suffisant pour féconder l'œuf est présent dans l'infundibulum au moment de l'ovulation, quel que soit l'état antérieur de la poule. Y a-t-il, dans le cas de poules en pause, stockage des spermatozoïdes dans les glandes infundibulaires, séjour prolongé des spermatozoïdes dans la lumière oviducale (les conditions sont favorables puisque les sécrétions liées à la formation de l'œuf sont inexistantes) ou bien migration en quantités faibles, mais non nulles, de spermatozoïdes provenant de la jonction utéro-vaginale ? Les observations des auteurs précités sont sans doute trop imprécises (peu d'animaux et grande variabilité individuelle) pour répondre à chacune de ces questions.

Quoi qu'il en soit, les travaux que nous venons d'évoquer indiquent que l'évacuation des spermatozoïdes provenant des glandes utéro-vaginales n'est ni sous contrôle hormonal, ni vraisemblablement dépendante du cycle de formation de l'œuf. Ceci conforte l'hypothèse avancée par Van Krey et al (1981) d'un "effeuillage" progressif des couches superficielles de spermatozoïdes stockés dans les glandes, qui pourrait être lié avec la diminution de leur capacité à s'agglutiner, un processus, semble-t-il, nécessaire à leur stockage.

\section{CONCLUSION}

De ce qui précède, nous pouvons à ce jour dégager les points suivants :

- La migration des spermatozoïdes, depuis l'entrée du vagin où ils sont déposés, jusqu'à la jonction utéro-vaginale où ils sont stockés, se réalise grâce à leur propre motilité : il s'agit donc d'un déplacement actif. Cette motilité n'est plus nécessaire entre la jonction utéro-vaginale et l'infundibulum (déplacement passif). Dans cette partie de l'oviducte, il est peu probable (mais non exclu) que les cils tapissant la lumière soient responsables de la migration. En revanche, les mouvements propres à l'oviducte, ainsi que le déplacement de fluide utérin pourraient être impliqués.

- Chez la poule, le stockage maximal n'est atteint que $2 \mathrm{j}$ environ après insémination intravaginale et non après quelques $h$ comme précédemment avancé. Chez la dinde, cette durée varie, selon les travaux, de 1 à $5 \mathrm{j}$. Ceci et le fait que de nombreux spermatozoïdes soient présents dans le vagin pendant les jours suivant l'insémination suggèrent que cette partie d'oviducte remplit un véritable rôle de stockage à court terme. Le déterminisme du stockage des spermatozoïdes ainsi que les conditions de leur survie prolongée dans les glandes de la jonction utéro-vaginale restent mal connus. II semble acquis cependant que l'agglutination et l'immobilisation temporaire dans les glandes utérovaginales soient nécessaires à leur survie prolongée dans cette zone. 
- Plusieurs observations indiquent que le stockage des spermatozoïdes dans les glandes utéro-vaginales se réalise en couches stratifiées. Mais la présence de nombreux spermatozoïdes dans la lumière vaginale et utérine dans les jours qui suivent l'insémination pose la question de leur possible participation à la fécondation des œufs par migration directe vers l'infundibulum.

- L'évacuation progressive des spermatozoïdes à partir des glandes utérovaginales ne semble ni sous contrôle hormonal ni directement dépendante du cycle de formation de l'œuf, ce qui conforte l'hypothèse d'un "effeuillage" continu des spermatozoïdes stockés dans la partie supérieure des glandes, peut-être par perte progressive de leur capacité à s'agglutiner.

\section{RÉFÉRENCES}

Allen TE, Grigg GW (1957) Sperm transport in ( the fow1. Aust J Agric Res 8, 788-799

Ashizawa K, Wishart GJ (1987) Resolution of the sperm motility stimulating principle of fow1 seminal plasma into $\mathrm{Ca}^{2+}$ and an unidentified low molecular weight factor. $J$ Reprod Fertil 81, 495-499

Bakst MR (1981) Sperm recovery from oviducts of turkeys at known intervals after insemination and oviposition. J Reprod Fertil 62, 159164

Bakst MR (1985) Zinc reduces turkey sperm oxygen uptake in vitro. Poult Sci 64, 564-566

Bakst MR (1988) Sperm release from uterovaginal junction sperm-storage tubules of the turkey : a histological assessment. Proc 11 th Int Cong Anim Reprod Artif Insem, Dublin, vol 3, 353

Bakst MR, Richards MP (1985) Concentration of selected cations in turkey serum and oviductal mucosae. Poult Sci 64, 555-563

Bilgili SF, Renden JA, McDaniel GR (1984) Relationship of fertility to day of insemination during the laying sequence. Poult Sci 63, 813-815
Bobr LW, Lorenz FW, Ogasawara FK (1964) Distribution of spermatozoa in the oviduct and fertility in domestic birds. II. Transport of spermatozoa in the fow1 oviduct. $J$ Reprod Fertil 8, 49-58

Brillard JP (1989a) Storage of spermatozoa in the utero-vaginal glands of hens as related with strain, dose, age and time after insemination. Proc 10th Ann Meet South Poult Sci Soc, Atlanta, 10

Brillard JP (1989b) Prediction of fertility from cell counts in the vitelline membrane of hen's eggs. Proc 10th Ann Meot South Poult Sci Soc, Atlanta, 9

Burke WH (1968) Release of spermatozoa from storage sites in the hen's oviduct. PhD Thesis, Univ of California, Diss Abstr 28, 4818B

Burke WH, Ogasawara FX (1969) Presence of spermatozoa in utero-vaginal fluids of the hen at various stages of the ovulatory cycle. Poult Sci 48, 408-413

Burke WH, Ogasawara FX, Fuqua CL (1969) Transport of spermatozoa to the site of fertilization in the absence of oviposition and ovulation in the chicken. Poult Sci 48, 602-608

Burke WH, Ogasawara FX, Fuqua CL (1972) A study of the ultrastructure of the utero-vaginal sperm storage glands of the hen, Gallus domesticus, in relation to a mechanism for the release of spermatozoa. J Reprod Fertil 29, 29-36

Bushman AF, Van Krey HP, Denbow DM, Siegel PB (1985) Effect of the ovulatory cycle on oviducal sperm storage in the domestic fow1. Theriogenology 23, 473-479

Christensen VL (1981) Effects of insemination intervals on oviducal sperm storage in turkeys. Poult Sci 60, 2150-2156

Compton MM, Van Krey HP (1979) Emptying of the utero-vaginal sperm storage glands in the absence of ovulation and oviposition in the domestic hen. Poult Sci 58, 187-190

Compton MM, Van Krey HP, Siegel PB (1978) The filling and emptying of utero-vaginal sperm host glands in the domestic hen. Poult Sci 57, 1696-1700

Crew FAE (1926) On fertility in the domestic fowl. Proc $R$ Soc Edinb Sect B 46, 230-238

Crichton EG, Krutzsch PH, Chvapil M (1982) Studies on prolonged spermatozoa survival 
in Chiroptera. II. The role of zinc of the spermatozoa storage phenomenon. Comp Biochem Physiol 7 1A, 71-77

EI Jack MH, Lake PE (1967) The content of the principal inorganic ions and carbon dioxide in uterine fluids of the domestic hen. $J$ Reprod Fertil 13, 127-132

Froman DP, Engel HN Jr (1989) Alteration of the spermatozoal glycocalyx and its effect on duration of fertility in the fow1 (Gallus domesticus). Biol Reprod 40, 615-621

Fujihara N, Koga O, Nishiyama H (1983) Possible participation of oviducal movement in spermatozoal transport in domestic fow1. Poult Sci62, 1101-1103

Garbers DL, Kopf GS (1980) The regulation of spermatozoa by calcium and cyclic nucleotides. Adv Cyclic Nucleotide Res 13, 251307

Grigg GW (1957) The structure of stored semen in the hen and the nature of the release mechanism. Poult Sci 36, 450-451

Hoover GN, Smith AH (1958) Secretion of fluid by the shell gland of the laying hen. Poult Sci 37, 467-471

Howarth B Jr (1971) Transport of spermatozoa in the reproductive tract of turkey hens. Poult Sci 50, 84-89

Mclntyre DR Jr (1984) Sperm storage in the oviduct of the turkey hen. PhD Thesis, Raleigh, USA

Mimura $H$ (1939) On the mechanism of travel of spermatozoa through the oviduct in the domestic fowl, with special reference to the arti- ficial insemination. Okajimas Folia Anat Jpn 17, 459-476

Mimura $H$ (1941) Studies on the mechanism of travel of spermatozoa through the oviduct in the domestic fowl. J Dept Agron, Univ Kyusyu 6, 167-259

Olsen MW, Neher BH (1948) The site of fertilization in the domestic fowl. $J$ Exp Zool 109, 355-356

Prasad S (1967) Histological location and role of utero-vaginal glands in storage and release of spermatozoa in chickens and turkeys. Ph D Thesis, University of Missouri, Columbia, Diss Abstr 28, 2114B

Schuppin GT, Van Krey HP, Denbow DM, Bakst MR, Meyer GB (1984) Ultrastructural analyses of utero-vaginal sperm storage glands in fertile and infertile turkey breeder hens. Poult Sci 63, 1872-1882

Van Krey HP (1964) Storage and transport of spermatozoa within the oviduct of the domestic fowl. Ph D, University of California, Davis. Diss Abstr 26, 2841

Van Krey HP, Balander RJ, Compton MM (1981) Storage and evacuation of spermatozoa from the utero-vaginal sperm host glands in domestic fowl. Poult Sci 60, 871-877

Verma OP, Cherms FL (1965) The appearence of sperm and their persistency in storage tubules of turkey hens after a single insemination. Poult Sci 44, 609-613

Verma OP, Cherms FL (1966) Recovery of spermatozoa from the reproductive tract of turkey hens. Poult Sci 45, 604-607 